\title{
A FIXED POINT THEOREM FOR POSITIVE $k$-SET CONTRACTIONS
}

\author{
by A. J. B. POTTER
}

(Received 16th April 1973)

\section{Introduction}

The abstract theory of positive compact operators (acting in a partially ordered Banach space) has proved to be particularly useful in the theory of integral equations. In a recent paper (2) it was shown that many of the now classical theorems for positive compact operators can be extended to certain classes of non-compact operators. One result, proved in (2, Theorem 5), was a fixed point theorem for compressive $k$-set contractions $(k<1)$. The main result of this paper (Theorem 3.3) shows that some of the hypotheses of (2, Theorem 5) are unnecessary. We use techniques based on those used by M. A. Krasnoselskii in the proof of Theorem 4.12 in (4), which is the classical fixed point theorem for compressive compact operators, to obtain a complete generalisation of this classical result to the $k$-set contractions $(k<1)$. It should be remarked that $\mathbf{J}$. D. Hamilton has extended the same result to $A$-proper mappings (3, Theorem 1). However apparently it is not known, even in the case when we are dealing with a $\Pi_{1}$-space, whether $k$-set contractions are $A$ proper or not.

In an attempt to justify our efforts we give a simple application of our result to prove the existence of a positive solution to an integral equation.

\section{Preliminaries}

For the sake of completeness we now define what we mean by a " $k$-set contraction". This involves the notion of the measure of non-compactness of a set.

Definition 2.1. Let $A$ be a bounded subset of a metric space $Y$. Then the measure of non-compactness of $A, \alpha_{Y}(A)$, is defined by

$\alpha_{\mathrm{Y}}(A)=\inf \{\varepsilon>0: A$ may be covered by finitely many sets of diameter $\leqq \varepsilon\}$.

It should be noted that $\alpha_{Y}(A)$ is independent of $Y$ in the following sense. If $Z$ and $Y$ are metric spaces and $A \subseteq Z \subseteq Y$ (the metric on $Z$ being that induced from $Y$ ) then $\alpha_{Z}(A)=\alpha_{Y}(A)$. With this in mind no confusion will result if the subscript of $\alpha_{Y}(A)$ is omitted. We will write $\alpha(A)$ for $\alpha_{Y}(A)$. The following proposition lists the main properties of the measure of non-compactness of a set. The proof can be found in (5, Chapter 1$)$. 
Proposition 2.2. Let $A$ and $B$ be subsets of the metric space $Y$. Then

(i) $A \subseteq B$ implies $\alpha(A) \leqq \alpha(B)$;

(ii) $\alpha(A \cup B)=\max \{\alpha(A), \alpha(B)\}$;

(iii) $\alpha(A)=\alpha(\bar{A})$.

If $Y$ is a Banach space, then

(iv) $\alpha(\operatorname{co}(A))=\alpha(A)$;

(v) $\alpha(A+B)=\alpha(A)+\alpha(B)$;

(vi) $\alpha(\lambda A)=|\lambda| \alpha(A)$;

(vii) $A$ is precompact implies $\alpha(A)=0$.

Note. Here and throughout the rest of the paper the following notation is used. If $A$ is a subset of a Banach space then $\bar{A}$ and $\operatorname{co}(A)$ are the closure and convex hull of $A$ respectively;

$$
\lambda A=\{\lambda a: a \in A\} \text { and } A+B=\{a+b: a \in A, b \in B\} .
$$

We now give the definition of $k$-set contractions.

Definition 2.3. An operator $T: Z \rightarrow Y$ ( $Z$ and $Y$ metric spaces) is said to be a $k$-set contraction if $T$ is continuous and $\alpha(T(A)) \leqq k \alpha(A)$ for all bounded subsets $A$ of $Z$.

The following proposition follows easily from this definition.

Proposition 2.4. Let $Y_{i}(i=1,2,3)$ be metric spaces and suppose

$$
T_{1}: Y_{1} \rightarrow Y_{2} \text { and } T_{2}: Y_{2} \rightarrow Y_{3}
$$

are $k_{1}, k_{2}$-set contractions respectively. Then $T_{2} T_{1}: Y_{1} \rightarrow Y_{3}$ is a $k_{1} k_{2}$-set contraction. If $S_{1}: Y_{1} \rightarrow X$ and $S_{2}: Y_{1} \rightarrow X$ (where $X$ is a Banach space), then $S_{1}+S_{2}: Y_{1} \rightarrow X$ is a $\left(k_{1}+k_{2}\right)$-set contraction.

There are many examples of $k$-set contractions. For instance operators of the form $H+C$, where $H$ is a $k$-contraction operating in a Banach space (i.e. $\|H x-H y\| \leqq k\|x-y\|$ for all $x, y$ in the domain of $H$ ) and $C$ is a compact operator (i.e. continuous and maps bounded sets into compact sets), are $k$-set contractions. In particular a compact operator is a 0 -set contraction.

The next proposition, which is the basis of the proof of the main theorem of this paper, was proved originally by Darbo (1).

Proposition 2.5. (The fixed point theorem for $k$-set contractions $(k<1)$ ). Let $C$ be a closed, convex, bounded subset of a Banach space $X$ and suppose $T: C \subset X \rightarrow C$ is a $k$-set contraction, $k<1$. Then there exists an $x \in C$ such that $T x=x$.

Note. We have used the notation $T: C \subset X \rightarrow C$ to imply that the metric on $C$ is that induced by the norm of $X$. In the rest of this paper the metric 
on a subset of a Banach space will always be assumed to be the one induced by the norm.

Before we proceed with the proof of our main theorem we give two simple lemmas.

Lemma 2.6. Let $D$ and $D^{\prime}$ be closed subsets of a metric space $Y$. Suppose $T: D \subset Y \rightarrow Y$ and $T^{\prime}: D^{\prime} \subset Y \rightarrow Y$ are $k$-set contractions such that $T=T^{\prime}$ on $D \cap D^{\prime}$. Define $T^{\prime \prime}: D \cup D^{\prime} \rightarrow Y$ by

Then $T^{\prime \prime}$ is a $k$-set contraction.

$$
T^{\prime \prime} x=\left\{\begin{array}{lll}
T x & \text { if } & x \in D \\
T^{\prime} x & \text { if } & x \in D^{\prime}
\end{array}\right.
$$

Proof. The continuity of $T^{\prime \prime}$ follows from a well-known lemma. The remainder of the proof follows directly from Proposition 2.2, (ii).

Lemma 2.7. Let $X$ be a Banach space, $T: D \subset X \rightarrow X$ a k-set contraction and $\lambda: D \rightarrow \mathbf{R}^{+}$a continuous function such that $\sup \{\lambda(x): \quad x \in D\}=l<\infty$. Define $T^{\prime}: \quad D \subset X \rightarrow X$ by $T^{\prime}(x)=\lambda(x) T x$ for all $x \in D$. Then $T^{\prime}$ is a $\mathrm{kl}$-set contraction.

Proof. $T^{\prime}$ is continuous. Let $A$ be a bounded subset of $D$. Then

$$
T^{\prime}(A) \subseteq \operatorname{co}(\{0\} \cup l T(A)) .
$$

By 2.2 (i), (ii), (iv) and (vi) we see that

This proves the lemma.

$$
\alpha(T(A)) \leqq l k \alpha(A) .
$$

\section{The fixed point theorem}

Throughout this section $C$ denotes a non-trivial cone in a Banach space $X$. ( $A$ subset $C$ of $X$ is a cone if $C$ is closed and

(i) $x, y \in C$ implies $\alpha x+\beta y \in C$ for all $\alpha, \beta \in \mathbf{R}^{+}$.

(ii) $\alpha x \in C$ and $-\alpha x \in C$ if and only if $x=0$.)

The cone $C$ induces a partial order on $X$ in the following manner. We say $x \leqq y$ if and only if $y-x \in C$. It is not difficult to verify that $\leqq$ induces a partial ordering on $X$. Note that $x \leq y$ means $y-x \notin C$.

It will be convenient to use the following notation. For $0<r<R$ let

$$
\begin{aligned}
& F_{r, R}=\{x \in C: r \leqq\|x\| \leqq R\} ; \\
& B_{r}=\{x \in C:\|x\| \leqq r\} ; \\
& S_{r}=\{x \in C:\|x\|=r\} .
\end{aligned}
$$

The next lemma is crucial in the proof of our theorem.

Lemma 3.1. Suppose $T: S_{r} \rightarrow C$ is a $k$-set contraction and let $T^{\prime}: B_{r} \rightarrow C$ 
be defined by

$$
T^{\prime} x= \begin{cases}(\|x\| / r) T((r /\|x\|) x) & \text { if } x \neq 0 \\ 0 & \text { if } x=0\end{cases}
$$

Then $T^{\prime}$ is a $k^{\prime}$-set contraction for $k^{\prime}$ as near $k$ as we please.

Proof. We first show that $T^{\prime}$ is continuous. Suppose the sequence $\left(x_{n}\right) \subset B_{r}$ is such that $x_{n} \rightarrow 0$ as $n \rightarrow \infty$ and $\left\|x_{n}\right\|>0$ for $n=1,2, \ldots$ Let $\lambda(x)=(\|x\| / r)$. Then

$$
\begin{aligned}
\left\|T^{\prime} x_{n}\right\| & =\left\|\lambda\left(x_{n}\right) T\left(\left(\lambda\left(x_{n}\right)\right)^{-1} x_{n}\right)\right\| \\
& \leqq \lambda\left(x_{n}\right) \sup \left\{\|T x\|: x \in S_{r}\right\} \\
& \rightarrow 0 \text { as } n \rightarrow \infty
\end{aligned}
$$

as $T\left(S_{r}\right)$ is bounded. It follows that $T^{\prime}$ is continuous at zero and thus it is not difficult to see that $T^{\prime}$ is continuous on all of $B_{r}$.

Let $A$ be a subset of $B_{r}$. If $\alpha(A)=0$ then $\bar{A}$ is compact by Proposition 2.2, (vii), and so $T^{\prime}(\bar{A})$ is compact. Hence $\alpha(T(A))=0$ and $\alpha(T(A)) \leqq k \alpha(A)$ trivially. Suppose $\alpha(A) \neq 0$. Choose $d>0$ such that

$$
k \alpha(A) \geqq 2 d>0
$$

(we may assume $k>0$; if not replace $k$ by $k+k^{\prime}$ for any $k^{\prime}>0$ ). Since $T^{\prime}$ is continuous there exists $\delta>0$ such that

$$
T^{\prime}\left(A \cap B_{\delta}\right) \subset B_{d}
$$

For any positive integer $n$ define $\varepsilon>0$ by $\delta=\varepsilon n$. Also define

Thus

$$
A_{m}=A \cap\{x:\|x\| \in[m \varepsilon,(m+1) \varepsilon]\} \text { for } m=0,1,2, \ldots
$$

Therefore

$$
A=\left(A \cap B_{\delta}\right) \cup\left(\bigcup_{m=n}^{N} A_{m}\right) \text { for some finite integer } N .
$$

$$
\begin{aligned}
\alpha\left(T^{\prime}(A)\right) & =\alpha\left(T^{\prime}\left(\left(A \cap B_{\delta}\right) \cup\left(\bigcup_{m}^{N} A_{m}\right)\right)\right) \\
& \leqq \max \left\{\alpha\left(T^{\prime}\left(A \cap B_{\delta}\right), \alpha\left(T^{\prime}\left(A_{n}\right)\right), \ldots\right\}\right.
\end{aligned}
$$

Let $D_{m}=B_{r} \cap A_{m}$. Then it follows from Lemma 2.7 that

$T^{\prime}: D_{m} \rightarrow X$ is a $(((m+1) / r) \varepsilon(r / m \varepsilon) k)$-set contraction,

i.e. $T^{\prime}: D_{m} \rightarrow X$ is a $k(1+(1 / m))$-set contraction.

Using (1), (2), (3) and (4) and Proposition 2.2,

$$
\begin{aligned}
\alpha\left(T^{\prime}(A)\right) & \leqq \max \left\{\alpha\left(B_{d}\right), k(1+(1 / n)) \alpha\left(A_{n}\right), k\left(1+(1 /(n+1)) \alpha\left(A_{n+1}\right), \ldots\right\}\right. \\
& \leqq \max \{k \alpha(A), k(1+(1 / n)) \alpha(A), \ldots\} \\
& \leqq k(1+(1 / n)) \alpha(A) .
\end{aligned}
$$

But we may choose $n$ as large as we please so the result follows. 
Definition 3.2. An operator $T: F_{r, R} \rightarrow C$ is said to be a compression of the cone $C$ if

and for all $\varepsilon>0$

$$
T x \leq x \text { for all } x \in C \text { with }\|x\|=r ;
$$

$$
T x \sum(1+\varepsilon) x \text { for all } x \in C \text { with }\|x\|=R \text {. }
$$

Remark. This is precisely the definition used by-Krasnoselskii in Chapter 4 of (4). It is more general than the definition used in (2).

We now give the promised fixed point theorem.

Theorem 3.3. Let $T: F_{r, R} \rightarrow C$ be a $k$-set contraction $(k<1)$, which compresses the cone $C$. Then $T$ has at least one nonzero fixed point in $C$.

Proof. We consider the operator $T^{\prime}: C \rightarrow C$ defined by

$$
T^{\prime} x= \begin{cases}\delta h & \text { if }\|x\|=0 ; \\ (\|x\| / r) T((r /\|x\|) x)+\delta h & \text { if } 0<\|x\| \leqq r-\delta \\ (\|x\| / r) T((r /\|x\|) x)+h(r-\|x\|) & \text { if } r-\delta \leqq\|x\| \leqq r \\ T x & \text { if } r \leqq\|x\| \leqq R \\ T((R /\|x\|) x) & \text { if } R \leqq\|x\| ;\end{cases}
$$

where $0<\delta<r, h \in C$ and

$$
\|h\|>(1 / \delta)\left[(r-\delta)+((r-\delta) / r) \sup \left\{\|T y\|: y \in S_{r}\right\}\right] ;
$$

(recall that $S_{r}=\{y \in C:\|y\|=r\}$ ).

Let $R^{\prime}=\sup \left\{\left\|T^{\prime} x\right\|: x \in B_{R}\right\}$ then $T^{\prime}: B_{R^{\prime \prime}} \rightarrow B_{R^{\prime \prime}}$ where $R^{\prime \prime}=\max \left(R^{\prime}, R\right)$. It follows from 2.4, 2.6, 2.7, 3.1 that $T^{\prime}$ is a $k^{\prime}$-set contraction for $k^{\prime}$ as near $k$ as we please; in particular we may choose $k^{\prime}<1$. As $B_{R^{\prime \prime}}$ is closed, convex and bounded, we may apply 2.5 and deduce that there exists $x^{*} \in C$ such that

$$
x^{*}=T^{\prime} x^{*} \text {. }
$$

We now show that $r \leqq\left\|x^{*}\right\| \leqq R$. It is impossible that $x^{*}=0$ so we assume

If (i) holds then

$$
\text { (i) } 0<\left\|x^{*}\right\| \leqq r-\delta \text {. }
$$

$$
x^{*}=\left(\left\|x^{*}\right\| / r\right) T\left(\left(r /\left\|x^{*}\right\|\right) x^{*}\right)+\delta h .
$$

Thus $h=(1 / \delta)\left[x^{*}-\left(\left\|x^{*}\right\| / r\right) T\left(\left(r /\left\|x^{*}\right\|\right) x^{*}\right)\right]$. Hence

$$
\|h\| \leqq(1 / \delta)\left[(r-\delta)+((r-\delta) / r) \sup \left\{\|T y\|: y \in S_{r}\right\}\right] .
$$

This contradicts our assumption on $h$. Next we assume

$$
\text { (ii) } r-\delta \leqq\|x\|<r \text {. }
$$

Then,

$$
\left.\left(r /\left\|x^{*}\right\|\right) x^{*}=T\left(\left(r /\left\|x^{*}\right\|\right) x^{*}\right)+\left(\left(r-\left\|x^{*}\right\|\right) / \| x^{*}\right)\right] r h .
$$

Thus $\left(r /\left\|x^{*}\right\|\right) x^{*} \geqq T\left(\left(r /\left\|x^{*}\right\|\right) x^{*}\right)$ which contradicts (5). Finally we assume (iii) $\left\|x^{*}\right\|>R$.

E.M.S. $-19 / 1-G$ 
Then

where

$$
T\left(\left(R /\left\|x^{*}\right\|\right) x^{*}\right)=(1+\varepsilon)\left(R /\left\|x^{*}\right\|\right) x^{*}
$$

$$
\varepsilon=\left(\left\|x^{*}\right\| / R\right)-1>0 .
$$

This contradicts (6) and so, as all other possibilities are excluded we have $r \leqq\left\|x^{*}\right\| \leqq R$ and our result follows.

Remark. We have based the proof of this theorem on the methods used by Krasnoselskii in the proof of Theorem 4.12 of (4). Using similar techniques it is also possible to generalise Theorem 4.14 of $(4)$ to $k$-set contractions $(k<1)$. The result we have obtained is the following.

Proposition 3.4. Let $T: F_{r, R} \rightarrow C$ be a $k$-set contraction $(k<1)$ which expands the cone $C$. Then $T$ has at least one non-zero fixed point in $C$. $\varepsilon>0$

(An operator $T: F_{r, R} \rightarrow C$ is said to be an expansion of the cone $C$ if for all and

$$
T x \geq(1+\varepsilon) x \text { for all } x \in C \text { with }\|x\|=r \text {; }
$$

$$
T x \leq x \text { for all } x \in C \text { with }\|x\|=R \text {.) }
$$

We omit the proof of this proposition as it is somewhat similar to the proof of 3.3 .

The main tool used in the proof of 3.3 was the fixed point theorem for $k$-set contractions $(k<1)$. This theorem can be extended to various classes of 1 -set contractions. The usual proof of this extension involves approximating to a 1 -set contraction by $k$-set contractions with $k<1$; then applying 2.5 to these approximants gives fixed points which (hopefully) converge to a fixed point of the 1-set contraction. Unfortunately this technique seems to fail when applied to 3.3 because the usual approximants do not preserve properties (5) and (6) of Definition 3.2. It would be interesting to know whether 3.3 can be extended to classes of operators larger than the class of $k$-set contractions $(k<1)$.

\section{Integral equations}

In this section we give an application of Theorem 3.3 to the theory of integral equations. We emphasise that there has been no attempt to achieve the best possible results as it is our intention to show how the theorem can be applied without obscuring the methods involved in a mass of technical details.

Let $\Omega$ be a closed subset of $\mathbf{R}^{n}$ and suppose that $X$ is the linear space of bounded continuous functions on $\Omega$. Endowed with the supremum norm $X$ becomes a Banach space. Let $k: \Omega \times \Omega \rightarrow \mathbf{R}$ be a continuous, bounded function and define the linear operator $K: X \rightarrow X$ by

$$
K x(s)=\int_{\Omega} k(s, t) x(t) d t, \quad x \in X
$$


We say that $K$ is the operator generated by the kernel $k$. It is well known that if $\Omega$ is bounded then $K: X \rightarrow X$ is a compact linear operator. However if $\Omega$ is unbounded and

$$
\sup \left\{\int_{\Omega}|k(s, t)| d t: s \in \Omega\right\}<\infty
$$

then, although $K$ is bounded, it is not necessarily compact, as is clearly demonstrated by the following example.

Suppose $\Omega=\mathbf{R}$ and $k(s, t)=k^{\prime}(s-t)$ where $k^{\prime}: \mathbf{R} \rightarrow \mathbf{R}$ is a continuous function with support contained in $[-1,1]$. Define the bounded sequence of functions $\left(x_{n}\right) \subset X$ by

$$
x_{n}(s)=x(s-n), \quad s \in \mathbf{R} ;
$$

where $x \in X$ has support contained in $[-1,1]$. Then,

where

$$
K x_{n}(s)= \begin{cases}0 & \text { if } s \in[n-2, n+2] \\ y_{n}(s) & \text { if } s \in[n-2, n+2]\end{cases}
$$

$$
\begin{aligned}
y_{n}(s) & =\int_{-\infty}^{\infty} k^{\prime}(s-t) x(t+n) d t \\
& =K x(s-n) .
\end{aligned}
$$

Obviously $\left(y_{n}\right)$ is a bounded sequence of functions in $X$ which has no convergent subsequence (provided $K x \neq 0$, a possibility quite easily avoided). Therefore $K$ is not compact.

However if

$$
\sup \left\{\int_{\Omega}|k(s, t)| d t: s \in \Omega_{n}\right\} \rightarrow 0 \text { as } n \rightarrow \infty
$$

(where $\Omega_{n}=\{s \in \Omega:|s| \geqq n\}$ ) then it can be shown that $K: X \rightarrow X$ (as defined by (7)) is compact. Hence it is simple to see how elementary examples of $k$-set contractions may arise from integral operators. Take, for example, $k=k_{1}+k_{2}$ where $k_{1}$ satisfies (2) and $k_{2}(s, t)=k_{2}^{\prime}(s-t)$ (as in our example). Then, if $K_{i}$ are the operators generated by $k_{i}(i=1,2)$, we see that $K=K_{1}+K_{2}$ is an $l$-set contraction with $l \leqq\left\|K_{2}\right\|$. (See the remarks immediately after Proposition 2.4.) It would be simple to give examples of $k_{1}$ and $k_{2}$ where $\left\|K_{2}\right\|<\|K\|$ and so our $l$-set contractions need not be " trivial" $l$-contractions.

It seems a difficult problem to calculate the $l$ appropriate to a particular $k$ precisely, therefore we will give this particular parameter as a hypothesis in our application; giving our result in terms of $l$ rather than more accessible properties of $k$.

From now on we assume that $k: \Omega \times \Omega \rightarrow \mathbf{R}^{+}$is a continuous bounded function which generates an $l$-set contraction in $X$. Let $f: \Omega \times \mathbf{R}^{+} \rightarrow \mathbf{R}^{+}$be a continuous, bounded function. Define the operator $F$ : $C \rightarrow C$ ( $C$ being the 
cone of non-negative functions in $X$ ) by

$$
(F x)(s)=f(s, x(s)), \quad x \in C
$$

We now give our long awaited application.

Theorem 4.1. Let $k$ and $f$ be defined as above and suppose the following additional conditions are satisfied.

(i) $\sup \left\{\int_{\Omega}|k(s, t)| d t: s \in \Omega\right\}=M<\infty$;

(ii) $\inf \{k(s, t): s \in \Omega\} \geqq \lambda \sup \{k(s, t): s \in \Omega\}$ for all $t \in \Omega$;

(iii) $f(s, t)$ is an increasing function in $t$ for each $s \in \Omega$ and is bounded for all $s \in \Omega, t \in[0, \infty)$;

(iv) there exists $r>0$ such that

and

$$
r-\lambda M \inf \{f(s, r): s \in \Omega\}<0
$$

$$
|f(s, x)-f(s, y)| \leqq \delta|x-y| \text { for all } s \in \mathbf{\Omega}
$$

and $x, y \in[r, \infty)$.

Then, if $\delta l<1$, there is a non-zero solution of the non-linear integral equation

$$
x(s)=\int_{\Omega} k(s, t) f(t, x(t)) d t .
$$

Proof. Let $K$ be the operator generated by $k$, and let

$$
\begin{aligned}
C & =\{x \in X: x(s) \geqq 0 \text { for all } s \in \Omega\} \text { and } \\
C^{\prime} & =\{x \in C: \inf (x(s): s \in \Omega) \geqq \lambda \sup (s(x): s \in \Omega)\} .
\end{aligned}
$$

Clearly both $C$ and $C^{\prime}$ are cones in $X$.

To prove this theorem it is enough to show that there is a non-zero solution to the operator equation $x=K F x$, where $F$ is defined by (9). Let $T=K F$. We will show that $T: C^{\prime} \rightarrow C^{\prime}$ is a $\delta l$-set contraction which compresses the cone $C^{\prime}$. As we have assumed that $\delta l<1$ our result will follow directly from 3.3.

We show initially that $K(C) \subset C^{\prime}$. (Note that our hypothesis that

$$
k: \Omega \times \Omega \rightarrow \mathbf{R}^{+}
$$

ensures that $K(C) \subset C$.) Suppose $x \in C$ then

$$
\begin{aligned}
\inf \left\{\int_{\Omega} k(s, t) x(t) d t: s \in \Omega\right\} & \geqq \int_{\Omega} \inf \{k(s, t): s \in \Omega\} x(t) d t \\
& \geqq \lambda \int_{\Omega} \sup \{k(s, t): s \in \Omega\} x(t) d t \quad \text { (by (ii)) } \\
& \left.\geqq \lambda \sup \int_{\Omega} k(s, t) x(t) d t: s \in \Omega\right\} .
\end{aligned}
$$


Let $D=\left\{x \in C^{\prime}:(r / \lambda) \leqq\|x\|\right\}$ where $r$ and $\lambda$ are defined in (ii) and (iv). The conditions $x \in C^{\prime}$ and $(r / \lambda) \leqq\|x\|$ imply that inf $\{x(s): s \in \mathbf{R}\} \geqq r$ and so it follows from (iv) that $F: D \rightarrow C$ is a $\delta$-contraction.

We have proved so far that $F: D \rightarrow C$ and $K: C \rightarrow C^{\prime}$, thus $T=K F: D \rightarrow C^{\prime}$ and as $F$ is a $\delta$-contraction and $K$ is an $l$-set contraction it follows from 2.4 that $T$ is a $\delta l$-set contraction. We need only prove that $T$ is a compression of $C^{\prime}$.

Suppose that $x \in C^{\prime}$ and $\|x\|=(r / \lambda)$. Then,

$$
\begin{aligned}
(x-T x)(s) & =x(s)-\int_{\Omega} k(s, t) f(t, x(t)) d t \\
& \leqq\|x\|-\int_{\Omega} k(s, t) f(t, \lambda\|x\|) d t
\end{aligned}
$$

(This follows from the definition of $C^{\prime}$ and condition (iii).) Hence

$$
(x-T x)(s) \leqq(r / \lambda)-\inf \{f(s, r): s \in \Omega\} \int_{\Omega} k(s, t) d t .
$$

Take $s_{0} \in \Omega$ such that $\int_{\Omega} k\left(s_{0}, t\right) d t>M-\varepsilon$ where $\varepsilon$ is small enough to allow

Then

$$
r-\lambda(M-\varepsilon) \inf \{f(s, r): s \in \Omega\}<0 \quad \text { (see (iv)). }
$$

and so

$$
(x-T x)\left(s_{0}\right)<0
$$

$$
x-T x \notin C .
$$

As $C^{\prime} \subset C$ it follows that

$$
x-T x \sum 0 \text { for all } x \in C^{\prime},\|x\|=(r / \lambda)
$$

where $\sum$ refers to the ordering induced by $C^{\prime}$.

Suppose $y \in C^{\prime}$. Then

$$
\begin{aligned}
(y-T y)(s) & =y(s)-\int k(s, t) f(t, y(t)) d t \\
& \geqq \lambda\|y\|-M M^{\prime} \text {, by (i), (ii) and (iii); }
\end{aligned}
$$

(where $M^{\prime} \geqq f(s, y)$ for all $s \in \Omega, y \in[0, \infty)$ ). Thus, if $\|y\|>\left(M M^{\prime} / \lambda\right)$ we see that

$$
y-T y \leq 0 \text { for all } y \in C^{\prime},\|y\|=R .
$$

(again $\leq$ refers to the ordering induced by $C^{\prime}$ ) where $R$ is some constant greater than $M M^{\prime} / \lambda$.

In view of (11) and (12) it follows that $T: F_{r / \lambda, R} \rightarrow C^{\prime}$ is a compression of the cone $C^{\prime}$ and so our theorem follows from 3.3.

The results of this paper form part of the author's thesis submitted to the University of Sussex in 1972. 


\section{REFERENCES}

(1) G. Darbo, Punti uniti in transformazioni a condominio non-compactto, Rend. Sem. Mat. Univ. Padova 24 (1955), 84-92.

(2) D. E. Edmunds, A. J. B. Potrer and C. A. Stuart. Non-compact positive operators, Proc. Roy. Soc. London, Ser. A, 328 (1972), 67-81.

(3) J. D. Hamilton, Non-compact mappings and cones in Banach Spaces, Arch. Rational Mech. Anal. 48 (1972), 153-162.

(4) M. A. Krasnoselski, Positive Solutions of Operator Equations (Groningen, 1964).

(5) R. D. Nussbaum, The fixed point index and fixed point theorems for $k$-set contractions (Unpublished Ph.D. thesis, Chicago, 1968).

\section{King's COLlege}

UNIVERSITY OF ABERDEEN 